\title{
AN ANALYSIS OF DESKTOP CONTROL AND INFORMATION RETRIEVAL FROM THE INTERNET USING AUGMENTED REALITY
}

\author{
Anup Raut ${ }^{1}$, Afroz Kazi ${ }^{2}$, Akshay Kamthe ${ }^{3}$, Sachin Waghmare ${ }^{4}$, Smita Kalunge ${ }^{5}$ \\ ${ }^{1}$ Assistant Professor, Department of Computer Engineering TCOER, Maharashtra, India \\ ${ }^{2}$ Research Scholar, Department of Computer Engineering TCOER, Maharashtra, India \\ ${ }^{3}$ Research Scholar, Department of Computer Engineering TCOER, Maharashtra, India \\ ${ }^{4}$ Research Scholar, Department of Computer Engineering TCOER, Maharashtra, India \\ ${ }^{5}$ Research Scholar, Department of Computer Engineering TCOER, Maharashtra, India
}

\begin{abstract}
As the use of computers is ever increasing new and easier methods of interaction with the system are needed. Augmented reality makes any application more interactive and full of life making it easier and attractive. The conventional mouse and keyboard can be replaced by human hand to interact with the computer. Addition of augmented reality to do so will make it more attractive. The same concept of using hand as interaction device and adding augmented reality can be used for retrieval of information from the internet. This will make our daily tasks relating computer easier and fun increasing productivity.
\end{abstract}

Keywords: Human Computer Interaction, Desktop Control, Information Access, Augmented Reality, Image Processing, Information Retrieval, Image Formation

\section{INTRODUCTION}

In today's time computers have become inseparable component to human beings. Even end users who have none or little technical knowledge interact with computers many times a day. Since the introduction of computers, the keyboard has been the primary input device. The introduction of mouse as a consumer peripheral in 1984 added another important input device to the computers. Since then a little has been changed in the way we interact with the computers. There is a need to replace the conventional ways of interaction with device that a human is more comfortable to use making it more attractive and intuitive. Addition of augmented reality will make interactions more realistic for a user.

\section{LITERATURE SURVEY}

Human Computer Interaction is the form of development of human interaction with computers. The HCI will further be developed to make the interactions more intuitive. Augmented Reality enhances the user experience of Human Computer Interaction. Augmented Reality is a combination of images generated by computer with real images. Augmented Reality deals with image processing. Various Image Processing algorithms are required in order to make Augmented Reality work. The use of human hand as a virtual object to interact with a computer makes an environment more user friendly. Natural hand movements can be used as a medium of communication with a machine making interactions practical and intuitive. Human centred designs and innovations are the primary considerations for designing of systems with larger life span and more productivity. Hence the system is proposed.

Various systems involving the use of Augmented Reality to make applications more real are used. The main objective of AR applications is to make the interactions natural and intuitive. AR enables the user to interact with the computer objects in a similar manner like real world objects.

Siegl et al. has introduced a concept of 3D cursors for interactions in the applications. The user just has to point with a finger or hand in the space towards the desired button. The camera will then capture the position and make the further processing.

Rafael Radkowski has presented a system that uses augmented reality for interactive assembly of $3 \mathrm{~d}$ models. The application tracks and monitors various movements and gestures using Microsoft Kinect video camera to take inputs from the user. It allows the user to interact and manipulate with the system.

Ching-Hao Lai has proposed various algorithms in his application for in vehicle computers that a driver uses in particular like GPS. The application taks inputs from users through various movements of hand and uses them to provide input the in vehicle system. The application aims at removing or reducing the risk of accidents that are caused due to distractions that are caused due to manual interaction of driver with in vehicle devices like GPS, music players etc. 


\section{PROPOSED SYSTEM}

We propose a system in which the user can interact with the computer without the use of conventional keyboard and mouse. The user makes use of his hands to give commands to the system. In order to make the interaction more intuitive and realistic the human hand is used. Humans are very comfortable in making interactions using human hand. Even a child can easily and effectively make use of his hands. Hence the hand can be used as a perfect device for interaction.

The system consists of two modules one for desktop control and other for information retrieval from the internet. The desktop control consists of various menus and buttons that a user frequently needs to access for example Media Player. User will see a Virtual menu on the desktop that will comprise of buttons to select from. Virtual menu is displayed on live captured feed for button selection.

The second module consists of daily information retrieval from the internet. We rely on the internet heavily for many of our activities. The application will allow users to access the information from the internet in a more realistic and intuitive manner making use of hand movements merged with augmented reality. Accessing information like news, stock updates. Weather forecast will become more real life than just some information displayed by a machine. User will feel like a part of the system as he himself will be interacting with the information without the presence of any device to mediate between the computer and the user.

\section{SYSTEM ARCHITECTURE}

The first action is performed with the help of a web cam. The camera captures the movements of the hand in the form of video feed. Frames derived from the video feed are used for further processing by the system. Then the retrieved frames are blurred using Gaussian Blur. It is used so that the image structures retrieved from image frames are enhanced at various scales. The blurred images are converted from RGB to HSV format (Gray Scale Conversion). The HSV format offers more accuracy than the RGB. It offers more distinct relation between the colours. Then the gray scale image i.e. the image in HSV format is converted into binary format by means of Thresholding. Thresholding converts the image in the form of only black and white i.e. only two value's 0 and 1 . The next step is blob detection. The Blobs from the black and white image are detected using blob detection algorithm. It does this by identifying group of white pixels. The position of the hand is recognized using image subtraction that calculates the position of the hand and processes the position to choose the selected menu. The position of the hand states the menu and a command is sent into the database to open the selected menu.

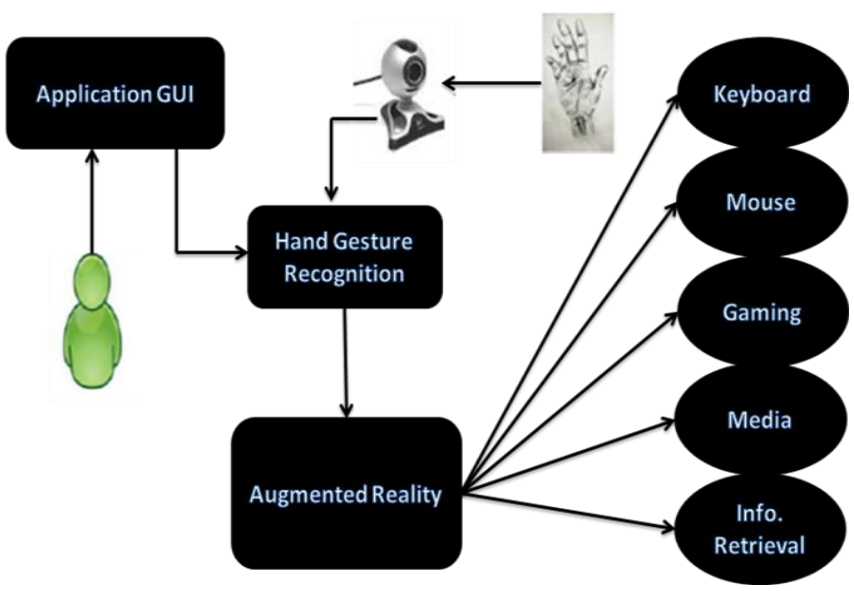

\section{CONCLUSION}

This paper proposes a system that will use the human hand as an input device to interact with the computer to replace the existing conventional input devices ie keyboard and mouse. The system aims at making the user interactions with the computer more realistic and intuitive. It shall allow users to control desktop by using their hands. Also it shall allow them to interact with the information on the internet by themselves without any mediatory device. Augmented reality makes the interactions more attractive and takes the user experience to another level. Augmented reality is already in the process of changing things as we know them. The proposed system uses this concept of Augmented Reality to entirely change the user experience and allow them flexibility and comfort in doing their daily interactions with the computer and the internet.

\section{ACKNOWLEDGEMENTS}

The authors wish to thank Department of Computer Engineering, TCOER. This work was supported in part by a grant from Mr. S.B. Choudhary(Head of the Department, Department of Computer Engineering, TCOER,Pune).

\section{REFERENCES}

[1] Siegl, H., Schweighofer, G., and Pinz, A., “An AR Human Computer Interface for Object Localization in a Cognitive Vision Framework". In: Int. Workshop on Computer Vision in HumanComputerInteraction (ECCV), Springer 3058, 2004, pp. 176-186

[2] Rafael Radkowski "Interactive Hand Gesture-based Assembly for Augmented Reality Applications" ACHI 2012 : The Fifth International Conference on Advances in Computer-Human Interactionsworth, pp. 123-135, 1993.

[3] Ching-Hao Lai "A Fast Gesture Recognition Scheme for Real-Time Human-Machine Interaction Systems" 2011 Conference on Technologies and Applications of Artificial Intelligence

[4] Yoki Ariyana and Aciek Ida Wuryandari, "Basic 3D Interaction Techniques in Augmented Reality", Center of Development and Empowerment Teachers and Education Personnel in Science Bandung, 
Indonesia,2012. Zhang, C. Zhu, J. K. O. Sin, and P. K. T. Mok, "A novel ultrathin elevated channel lowtemperature poly-Si TFT," IEEE Electron Device Lett., vol. 20, pp. 569-571, Nov. 1999.

[5] Ronald Azuma, Yohan Baillot and Steven Feiner," Recent Advances in Augmented Reality", Columbia University,2010

[6] Fetaji, Majlinda.,Loskoska, Suzana., FetajiBekim,EbibiMirlinda., "Investigating Human Computer Interaction Issues In Designing Efficient Virtual Learning Environments", South East European University,2007.M. Shell. (2002) IEEEtran homepage on CTAN. [Online]. Available: http://www.ctan.org/tex-

archive/macros/latex/contrib/supported/IEEEtran/

[7] Lee, T. and Höllerer, T., "Hybrid Feature Tracking and User Interaction for Markerless Augmented Reality," in Proceedings of IEEE Virtual Reality 2008, 8-12 March, Reno, Nevada, USA, 2008, pp. 145-152 "PDCA12-70 data sheet," Opto Speed SA, Mezzovico, Switzerland.

[8] Hackenberg, G., McCall, R., and Broll, W., „Lightweight Palm and Finger Tracking for RealTime 3D Gesture Control.“ in IEEE Virtual Reality 2011, pp. 19-26, Singapore, 2011

[9] Vance, J. and Dumont, G., „A Conceptual Framework to SupportNatural Interaction for Virtual Assembly Tasks,“ in Proc. Of theASME 2011 World Conference of Innovative Virtual Reality,WINVR2011, Jun3 27-29, Milan, Italy, 2011, pp. 1-6

[10] Shen, Q., „A Method for Composing Virtual Prototypes ofMecha-tronic Systems in Virtual Environments, “ Ph.D. Thesis,University of Paderborn, HNI-Verlagsschriftenreihe, Paderborn, Vol.193, Dez. 2006Nayak, G. C., and Zienkiewicz, O. C., 1972, Convenient forms of stress invariants for plasticity, Proc. ASCE, 98(4), 949-953.

\section{BIOGRAPHIES}

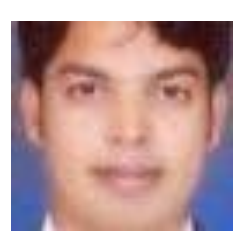

Anup Raut Assistant Professor of Department of Computer Engineering, Trinity College of Engineering and Research, Pune, INDIA. Contact Details: +91 $9960747450 \quad$ Email-ID anupraut25@gmail.com

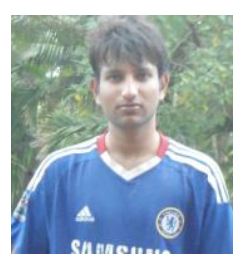

Afroz Kazi, Research Scholar, Student of Final Year of Department of Computer Engineering, Trinity College of Engineering and Research, Pune, INDIA. Contact Details: +91 9028772567 EmailID aj1343@gmail.com 